\title{
Primary protrusio acetabuli in childhood
}

\author{
Krupa Shah $\cdot$ Hitesh Shah
}

Received: 8 September 2010 /Revised: 24 September 2010 /Accepted: 29 September 2010 /Published online: 10 October 2010

(C) Springer-Verlag 2010

An 11-year-old girl presented with a waddling gait and restricted abduction of both hips. A pelvic radiograph shows bilateral deepening of the acetabular cavity (Fig. 1). All other radiographs were normal. Syndromes like Marfan, Stickler, Beals, Kniest and Ehlers-Danlos were ruled out. Complete blood counts, calcium, phosphorus, alkaline phosphatase, rheumatoid factor and antinuclear antibodies were within normal limits.

A center-edge angle greater than $40^{\circ}$ and medialization of the medial wall of the acetabulum past the ilioischial line are the diagnostic features for protrusio acetabulum in an anteroposterior pelvic radiograph [1]. The protrusio acetabulum is caused by a primary idiopathic disorder and secondary infectious, metabolic, inflammatory, traumatic, neoplastic and genetic disorders [2]. Primary protrusio acetabulum is common in females, bilaterally, and in adolescents. The triradiate cartilage closure is effective in reversing or stopping the protrusio before 10 years of age [1]. Early diagnosis and treatment reduce long-term disability and secondary osteoarthritis in patients.

K. Shah

Department of Obstetrics and Gynecology, Kasturba Medical

College, Manipal University,

Manipal, India

\section{H. Shah $(\bowtie)$}

Division of Pediatric Orthopedics, Department of Orthopedics,

Kasturba Medical College, Manipal University,

Manipal, India 576104

e-mail: hiteshshah12@gmail.com

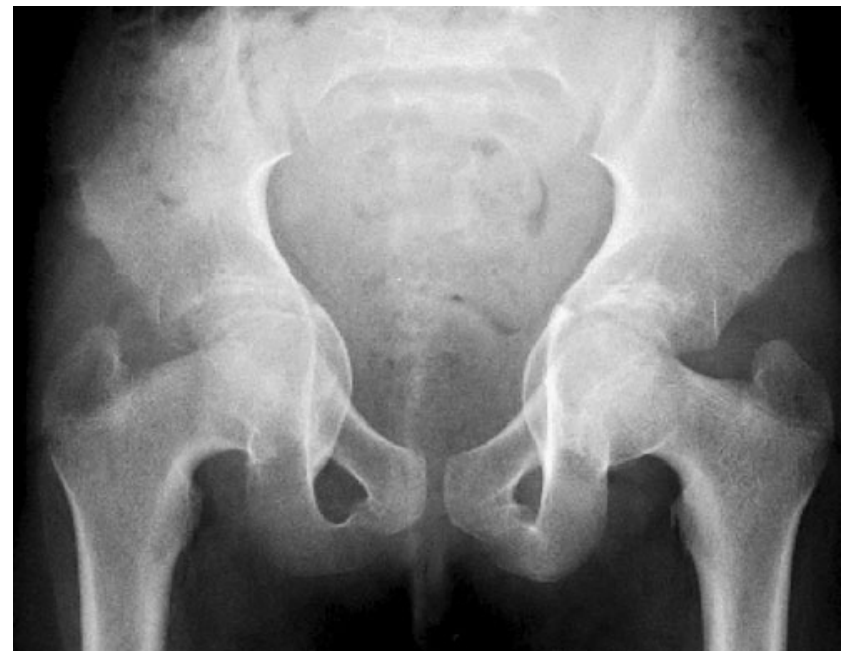

Fig. 1 Pelvic radiograph

\section{References}

1. Steel HH (1996) Protrusio acetabuli: its occurrence in the completely expressed Marfan syndrome and its musculoskeletal component and a procedure to arrest the course of protrusion in the growing pelvis. J Pediatr Orthop 16:704-718

2. Van De Velde S, Fillman R, Yandow S (2006) The aetiology of protrusio acetabuli. Literature review from 1824 to 2006. Acta Orthop Belg 72:524-529 